\title{
¿Es necesaria la orquidectomía en los pacientes con testículo no descendido post-puberal?
}

\author{
E.A. Granados Loarca, S. Esau Ortega \\ Servicio de Urología. Hospital General de Enfermedades Zona 9. \\ Instituto Guatemalteco de Seguridad Social. Guatemala.
}

Actas Urol Esp 2005; 29 (10): 969-973

\section{RESUMEN \\ ¿ES NECESARIA LA ORQUIDECTOMÍA EN LOS PACIENTES CON TESTÍCULO NO DESCENDIDO POST-PUBERAL?}

Ojetivo: La criptorquidia es un factor de riesgo de cáncer de testículo, y se ha asociado con cáncer en el testículo contralateral. Los mecanismos por lo cual incrementa el riesgo de cáncer en el testículo criptorquídico no está claro.

Material y Métodos: Se analizaron 25 pacientes mayores de 20 años que acudieron por Testículo no Descendido (TND); a los cuales se les practicó orquidectomía en 22 testículos y orquidopexia en 7 testículos. El tiempo que los pacientes tuvieron el/los testículos en localización anómala, osciló entre 20-51 años.

Resultados: Los pacientes mayores con TND suelen acudir por no palparse el testículo en el escroto, y el $16 \%$ por infertilidad. Sólo en $1 / 25$ casos $(4 \%)$ se demostró cáncer que fue un seminoma puro.

Conclusiones: En el seguimiento de los pacientes, ninguno presentó cáncer en el testículo contralateral entre 5-10 años. En los pacientes en los que se efectuó orquidopexia ninguno presentó cáncer del testículo entre 5-11 años.

Palabras clave: Cáncer. Testículo no descendido.

\section{ABSTRACT}

\section{IS NECESSARY TO PRACTICE ORCHIECTOMY IN PATIENTS WITH POST-PUBERAL} MALDESCENDED TESTES?

Objective: The cryptorchidism is a risk factor for testicular cancer and is associated with cancer in the contraleteral testicle. The mechanism that leads to an increased risk of causes in a cryptorchid testis is unclear.

Materials and Methods: We analyzed 25 patients of 20-years old for maldescended testicles we practiced orchiectomy in 22 testicles and orchiopexy in 7 testicles. The time patients had the testicles in an anomalous location were between 20-51 years.

Results: The elder patients with maldescends testicles came because the couldn't feel the testicle in the scrotum and the $16 \%$ of them because of infertility. Only 1 of 25 cases (4\%) were demonstrated cancer with pure seminoma.

Conclusions: In the following up of the patients none of them presented contralateral testicle cancer between 5-10 years. The patients who were practiced the orchiopexy no-one presented testicular cancer between 5-11 years.

Keywords: Cancer. Maldescended testes.

$\mathrm{S}_{\mathrm{e}}^{\mathrm{e}}$ ha informado que en los pacientes con tesículo no descendido (criptorquidia) aumenta la frecuencia de cáncer en el testículo, por lo que se ha recomendado la orquidectomía de los testículos no descendidos ${ }^{1,6}$ en especial en los que están a nivel abdominal, ya que el 30\% de los abdominales suelen presentar tumor en el testículo ${ }^{21}$. Presentamos una serie de 25 pacientes mayores de 20 años, que fueron tratados por testículo no descendido. 


\section{MATERIAL Y MÉTODOS}

Se efectuó un estudio retro y prospectivo, en 25 pacientes mayores de 20 años que acudieron por Testículo no Descendido (TND), a quienes se practicó orquidectomía en 22 testículos y orquidopexia en 7 testículos. Se tomaron los siguientes parámetros: La edad, la cual osciló entre 20-51 años (media 27,8\%). Los antecedentes, 3 pacientes habían sido operados de TND en el mismo lado, y un paciente había sido operado de hernia inguinal derecha. $\mathrm{El}$ motivo de consulta. TND en 23 casos, dolor testicular en 9 casos, e infertilidad en 4 casos. En cuanto a la localización, 9 casos eran derechos, 11 casos izquierdos y 5 fueron bilaterales. Veintidós testículos se palparon a nivel de la ingle, 4 a nivel del pubis, y 4 testículos no se palparon, deduciéndose que se encontraban a nivel intra-abdominal. Al tacto, se consideraron 6 como testículos normales y 20 como testículos pequeños. En todos los casos se efectuaron marcadores HCG y alfa-feto proteína. La exploración se efectuó, en 24 casos por vía inguinal, con el propósito de verificar si el testículo se encontraban en cualquier lugar del canal inguinal o por el mejor acceso de los que se encontraban a nivel intra-abdominal, cerca del anillo inguinal, y en un caso se efectuó vídeo-laparoscopia. Se encontró el testículo a nivel inguinal en 26 casos y en 4 casos a nivel intra abdominal. Cuatro pacientes tenían hernia inguinal en el mismo lado del TND. Seis casos fueron considerados normales, 20 pequeños y un caso indurado. La elección en la decisión de efectuar orquidectomía u orquidopexia dependió del criterio del cirujano. En 23 testículos se efectuó orquidectomía, sin importar el tamaño y localización del testículo, que fueron 8 derechos y 15 izquierdos; y en 7 testículos se practicó orquidopexia. Estos tenían un tamaño entre $2-5 \mathrm{~cm}$ de longitud y entre 1 y $3 \mathrm{~cm}$ de ancho, medidos con pie de rey; de ellos, 3 derechos y 4 izquierdos. En un paciente con TND bilateral se efectuó orquidectomía en un testículo de $2 \times 1,5 \mathrm{~cm}$ y biopsia contralateral.

La edad en que el paciente o la familia se dieron cuenta del (TND) fue de 5-45 años. Y el tiempo que los pacientes tuvieron el/los testículos en localización anómala, osciló entre 51-20 años.

En 15/20 casos en los que se efectuó seminograma, existían alteraciones en el seminograma, previo a la operación.

\section{RESULTADOS}

Los pacientes mayores con TND suelen acudir a consulta la mayor parte de las veces por no palparse el testículo en el escroto, y un 16\% por infertilidad; ocurriendo de forma más frecuente en el lado izquierdo. En el caso que nos ocupa, la exploración se efectuó, a excepción de un caso, por la vía inguinal; encontrándose el testículo en la mayoría de los casos en la ingle. En los testículos intraabdominales se logró su localización, y descenso hasta llevarlos al anillo inguinal, lo cual se puede efectuar a igual que la orquidectomía por vídeo-laparoscopia. En los pacientes con TND bilateral, en los que se encontró uno o ambos testículos pequeños, se efectuó orquidectomía y biopsia contralateral en el más grande.

La anatomía patológica informó: en los testículos pequeños atrofia, tejido fibroso, ausencia de células germinales, hiperplasia células de Leydig, fibrosis intersticial y atrofia tubular. Sólo en 1/15 casos $(4 \%)$ y $1 / 22(4,5 \%)$ de los testículos en quienes se practicó orquidectomía se demostró cáncer, que fue un seminoma puro. El paciente que desarrolló el seminoma puro confinado al testículo sin afectar el cordón estadio I, era un paciente de 28 años en el momento de su diagnóstico que acudió a consulta por no palparse el testículo derecho. Los marcadores alfa-feto proteína fueron normales y la HCG fue negativa. En la exploración inguinal se observó que se encontraba en el anillo inguinal intra-abdominal con un tamaño de $2,5 \times 1,5 \mathrm{~cm}$, duro, por lo cual se decidió la orquidectomía. El seguimiento de los pacientes, tanto en los que se efectuó orquidectomía $\mathrm{u}$ orquidopexia, se efectuó mediante la palpación del testículo y con ultrasonido dos veces por año (cada 6 meses) durante 5 años y luego una vez por año. En 2 casos en los que se efectuó orquidopexia, se practicó biopsia de ambos testículos por problema de infertilidad y se aprovechó la muestra para su evaluación. En los primeros 5 años se les efectuó marcadores alfa-feto proteína y Gonadotropina Coriónica, las cuales fueron normales, en especial en el caso del seminoma puro.

Ninguno de los pacientes a los que se efectuó orquidectomía presentó cáncer en el testículo contralateral entre 5-10 años. Ninguno de los pacientes en los que se efectuó orquidopexia presentó cáncer del testículo entre 5 y 11 años. 
En 20/25 casos se efectuó seminograma postoperatorio y en $7 / 20$ persistían alteraciones en el seminograma a 5 años de la cirugía. Se observó que conforme se aleja el testículo del escroto aumenta la posibilidad de infertilidad.

\section{DISCUSIÓN}

El tumor testicular representa el 1-2\% de todas las neoplasias del sexo masculino, y el $4 \%$ de los urogenitales ${ }^{8}$. Es el tumor sólido más frecuente en varones jóvenes entre los 20-40 años ${ }^{7,8}$.

El 86\% de todos los testículos están descendidos normalmente en el momento del nacimiento, el resto desciende en el transcurso del primer año de vida $^{4,5}$. La criptorquidia constituye la patología más común de la diferenciación sexual, con una incidencia $0,8-5,8 \%$ en niños nacidos a término $^{2,7,10}$. La incidencia en el prematuro es mucho mayor, hasta $20-30 \%{ }^{9}$. El lado más afectado varía según las series ${ }^{7,9,14}$, en la nuestra fue el testículo izquierdo.

La criptorquidia ha sido definida como un factor de riesgo para la transformación maligna, o disfunción de las células de Leydig y/o de Sertoli del testículo isolateral o contralateral ${ }^{1,6,12}$. Algunos autores lo calculan de 4-5 veces, y otros de 9-50 veces mayor que en el testículo nor$\mathrm{mal}^{4,5,7,9,11}$. El 3,5-12,9\%6-8,10,18 de los pacientes con tumor testicular tienen historia de criptorquidia, y de éstos el $25 \%$ se desarrollan en el testículo normal.

En el testículo no descendido ya se detectan cambios a partir de los 2 primeros años de vida; siendo las principales complicaciones la infertilidad, la torsión y la malignación ${ }^{2}$. Existen hipótesis del porqué de la malignización ${ }^{2}$, entre ellas se mencionan: alteraciones hormonales ${ }^{17}$, temperatura elevada, virus ${ }^{1}$, interferencia con el aporte sanguíneo o factores genéticos.

Entre los medios diagnósticos, Weiss et al. ${ }^{22}$ describieron que el ultrasonido, identifica el $70 \%$ de los testículos palpables, y que sólo identificaron $8 / 21$ de los no palpables, con lo que concluyen que el ultrasonido solo no puede utilizarse de forma satisfactoria, como método de exploración en el manejo de los testículos no descendidos ${ }^{2}$.

En nuestra serie hubo más problema en identificar los testículos intra-abdominales. Se obser- vó que conforme aumenta la distancia del testículo al escroto, la ecogenicidad de los testículos tiende a ser más hipoecoica. El eco-doppler es de mayor utilidad que el ultrasonido estándar. La tomografía es el medio de diagnóstico de elección no invasivo en el TND o no palpable ${ }^{15}$ y la vídeo laparoscopia, nosotros la efectuamos en los testículos intra abdominales.

La incidencia de criptorquidia en el hombre adulto es del 0,3-04\%, representando el 20\% de los testículos que se encuentran en el abdomen o pelvis ${ }^{4,15}$ y de estos, no todos presentan tumores del testículo. Villavicencio et al. ${ }^{21}$ informaron que sólo el $15 \%$ de los inguinales, y el 30\% de los abdominales presentaban tumor del testículo. Lo que indica que el ritmo de malignidad incrementa con la distancia del testículo al escroto ${ }^{15}$.

Respecto al tipo de tumores ${ }^{4,7,13}$, el tumor más frecuente en pacientes con criptorquidia no corregida es el seminoma (60-64\%). Halme et al. ${ }^{11}$ informaron que en los testículos abdominales el tumor más frecuente es el seminoma puro $(88 \%)$ y en menor proporción el carcinoma embrionario $^{5}$. En los tumores inguinales (64\%) se mantiene el seminoma puro, el carcinoma embrionario y en menor proporción el teratocarcinoma. En los tumores escrotales aumenta el teratocarcinoma, seguido del carcinoma embrionario, seminoma puro y coriocarcinoma puro $^{9}$.

Batata et $\mathrm{al}^{4}$ reportaron que la mayor incidencia de los seminomas ocurre en los testículos escrotales. Babaian y Zagars ${ }^{3}$ informaron que los tumores testiculares en pacientes con criptorquidia, en su mayoría suelen encontrarse en estadio II.

Un aspecto importante es si la orquidopexia reduce el riesgo de cáncer de testículo. Sobre esto existen distintas opiniones, Cedron ${ }^{7}$ y Jones $^{13}$ refieren que la orquidopexia no reduce el riesgo y que en ellos aumenta el predominio de tumores no-seminomatosos (78\%). Giwercman ${ }^{9}$ y Halms $^{11}$ reportaron que no aumenta el riesgo, sino al contrario reduce el riesgo y la consecuente infertilidad.

Otro factor importante es la presencia de carcinoma in situ (CIS) en los testículos no descendidos, ya que se ha reportado una incidencia de 1:1500; o del 2,8-25\% en los testículos no descendidos abdominales, y del 0,68\% en los inguinales, y del $0,1-5 \%$ después de la orquidopexia ${ }^{7,19}$. 
Pedersen et $\mathrm{al}^{18}$ demostraron CIS en $4 / 50$ pacientes con criptorquidia (8\%), e informaron que $3 / 94$ biopsias bilaterales tuvieron Carcinoma in situ, $\mathrm{y}$ no hubo correlación entre la localización de los testículos. Giwercman et al. ${ }^{10}$ sólo tuvieron 5 carcinomas in situ en 300 biopsias. El tratamiento del CIS uni o bilateral asociado a infertilidad o a criptorquidia es la orquidectomía. En nuestra serie no se observó ningún caso de CIS.

El pronóstico del cáncer de testículo ha cambiado. En la población danesa el riesgo es del $0,5 \%$, aumentando el riesgo cuando existe criptorquidia bilateral a un $9,3 \%$, a diferencia del testículo no descendido unilateral de $2-5 \%{ }^{9}$ porcentaje parecido al de la población general 0,5-2\%.

Existe entonces el dilema de qué hacer con un paciente después de la pubertad con un testículo no descendido. ¿Es cierto que en los testículos no descendidos la frecuencia de cáncer puede ser hasta de 50 veces más? ¿Es necesaria la Orquidectomía en todos los pacientes con criptorquidia? o ¿Es mejor practicar biopsia del testículo no descendido y orquidopexia? Cendron y Duckett ${ }^{7}$ prefieren la Orquidectomía y la colocación de prótesis. Wrighr ${ }^{23}$ refiere efectuar la fijación contralateral posterior a la Orquidectomía, especialmente si se encuentra un testículo atrófico, ya que no está claro si el testículo es agenésico o si su desaparición es consecuencia de una torsión. Solé Balcells ${ }^{20}$ es partidario de practicar la orquidopexia. Hay quienes prefieren efectuar biopsia de testículos y si existe CIS efectuar Orquidectomía $^{16,18,19}$.

Castiñeiras et $\mathrm{al}^{6}$ informaron que de 72 biopsias en testículos criptorquídicos ninguno manifestó tumor. Batata et $\mathrm{al}^{5}$ informaron que de 1.152 pacientes con criptorquidia 137 manifestaron cáncer. Von der Maase et $\mathrm{al}^{16}$ informaron una serie de 50 pacientes con criptorquidia a quienes se les efectuó biopsia, y sólo el $8 \%$ fue positivo a CIS.

En nuestra serie $1 / 25$ pacientes desarrollaron cáncer. En una revisión que efectué en mi fase de residencia en la Fundación Puigvert, en 62 casos revisados, en los que se practicó orquidectomía por TND sólo hubo 3/62 (4,8\%) casos de cáncer, 2 casos de Ca. embrionario y 1 caso de seminoma. ¿No hubiese sido mejor practicar la orquidopexia en estos casos y un seguimiento adecuado basado en la clínica y ultrasonido ahora que el pronóstico del tumor testicular ha mejorado, $\mathrm{y}$ dejar la orquidectomía para los testículos abdominales o para los que tengan CIS o Ca?.

Creemos que el manejo de los pacientes con TND debe ser de la siguiente manera:

Si el paciente tiene sólo un testículo, independiente del tamaño, éste debe de fijarse mediante orquidopexia y seguimiento adecuado. $\mathrm{Si}$ el paciente tiene dos testículos y tiene un TND, debe de fijarse por orquidopexia, si no está intra-abdominal y es mayor de $0,5 \mathrm{~cm}$. Si tiene los dos TND deben de fijarse los dos, si tiene un tamaño mayor de $0,5 \mathrm{~cm}$, dejando la orquidectomía para sólo un testículo si está intra-abdominal y si tiene menos de $0,5 \mathrm{~cm}$ de longitud.

\section{CONCLUSIONES}

Los pacientes mayores con TND suelen acudir en la mayoría de los casos, por no palparse el testículo en el escroto, y un 16\% por infertilidad.

En estos casos es mejor la exploración por la vía inguinal. $\mathrm{Y}$ en los casos intra-abdominales se puede utilizar la vídeo-laparoscopia.

En la histología de los TND suele observarse atrofia, tejido fibroso, ausencia de células germinales, hiperplasia de células de Leydig, fibrosis intersticial, y atrofia tubular.

El seguimiento de los pacientes debe hacerse con la clínica (tacto), ultrasonido y marcadores.

El pronóstico del cáncer de testículo ha cambiado. El porcentaje de afectación por cáncer el TND es parecido al de la población en general.

No en todos los casos de TND debe de efectuarse orquidectomía.

La orquidopexia en los TND no predispone a cáncer.

\section{REFERENCIAS}

1. Algood CB, Newell GR, Johnson DE. Viral etiology of testicular tumors. J Urol. 1988;139(2):308-310.

2. Arnaiz JF, Pérez JA, Fernández J, et al. Carcinoma embrionario en testículo criptorquídico: a propósito de un caso. Arch Esp Urol 1992;45:156.

3. Babaian RJ, Zagars GK. Testicular seminom: the MD Anderson experience. An analysis of pathological and patient characteristics, and treatment recommendations. J Urol 1988;139:311-314.

4. Batata MA, Whitmore WF, Chu FCH, Hilaris BS, Loh J, Grabstald H, Golbey R. Criptorchidis, and testicular cancer. J Urol 1980;124:382-387.

5. Batata MA, Chu FCH, Hilaris BS, Whitmore WF, Golbey RB. Testicular cancer in criptorchids. Cancer 1982;49:10231030. 
6. Castiñeiras J, López A,Vilches J, Cabello P, Rodríguez Rubio F. Criptorquidismo, orquidopexia y cáncer de testículo: aportación de un caso de esta asociación. Arch Esp Urol 1988;41:886.

7. Cedron M, Duckett JW. The maldescended testicle. AUA update series 10. Lesson 1991;28:218.

8. Gil MJ, Caro C, Villanueva A, et al. Manejo diagnóstico y terapéutico de los tumores testiculares. Revisión de quince años. Arch Esp Urol 1991;44:417.

9. Giwercman A, Grindsted J, Hansen B, Hansen OM, Skakkebaek NE. Testicular cancer risk in boys with maldescended testis: a cohort study. J Urol 1987;138:1214-1216.

10. Giwercman A, Bruu E, Frimodt-Moller CF, Skakkebaek NE. Prevalence of carcinoma in situ and other histophatological abnormalities in testes of with a history of cryptorchidism. J Urol 1989;142:998-1001.

11. Halme A, Kwllokumpu-Lehtienen P, Lehtonen T, Teppo L. Morphology of testicular germ cell tumours in trated and untreated cryptorchidism. Br J Urol 1989;64:78-83.

12. Halme A O, Huhtaniemi I, Wahlstrom T. Determinación de gonadotropinas testiculares y sus receptores en testículos criptorquídicos humanos mediante inmunohistoquímica y análisis de radio receptores. Act Endocrinol 1982;3:128.

13. Jones BJ, Thornhill JA, O'Donnell B, Kelly DG, Walsh A, Fennelly JJ, et al. Influence of prior orchiopexy on stage and prognosis of testicular cancer. Eur Urol 1991;19:201203

14. Kogan SJ, Gill B, Bennett B, Smey P, Reda EF, Levitt SB. Monorquidismo humano: estudio clinicopatológico de la ausencia testicular unilateral en 65 niños. J Urol 1986; 135:758.

15. Lorigan JG, Shirkhoda A, Dexeus FH. MR imaging of malignant germ cell tumour of the undescended testis. Urol Radiol 1989;11:113-117.
16. Von der Maase HV, Giwercman A, Müller J, Skakkebaek NE. Management of carcinoma -in situ- of the testis. Int $\mathrm{J}$ Androl 1987;10:209-220.

17. Moss AR, Osmond D, Bacchetto P, Torti FM, Gurgin V. Hormonal risk factors in testicular cancer. Am J Epidemiol 1986; 124:39-52.

18. Pedersen KV, Boiesen P, Zetter-Lund CG. Experience of screening for carcinoma -in situ- of the testis among young men with surgically corrected maldescended testes. In J Androl 1987;10:181-185.

19. Reinberg Y, Manivel JC, Fraley EE. Carcinoma in situ of the testis. J Urol 1989;142:243-247.

20. Solé Balcells F. Auvert J, Mathe G, Pavone M, Pérez S, Radaban M. Mesa redonda sobre "Pautas terapéuticas en los tumors de testículos" VI Curso de Urología. C.S. "La Paz" Madrid, 1975.

21. Villavicencio H, Martínez E, Vicente-Carretero R. Tumor testicular bilateral simultáneo. Actas Urol Esp 1989;13: 403-404.

22. Weis SM, Cárter AR, Rosenfield AT. Ultrasonografía de alta resolución para la localización de testículos no descendidos. J Urol 1986;135:936.

23. Whight JE. Testículo impalpable: Revisión de 100 niños. J Pediatr Surg 1986;21:151.

Dr. E.A. Granados Loarca

31 Avda. 14-60 Zona 7

Cond. Villas San Martín, 26

Guatemala

(Trabajo recibido el 30 marzo 2005) 